\title{
BMJ Open AMEND study protocol: a case-control study to assess the long-term impact of invasive meningococcal disease in Australian adolescents and young adults
}

\author{
Helen Marshall (D) ,, Mark McMillan (D) ,, ${ }^{1,2}$ Bing Wang, ${ }^{1,2}$ Robert Booy, ${ }^{3,4}$ \\ Hossein Afzali, ${ }^{5}$ Jim Buttery, ${ }^{6}$ Christopher C Blyth, ${ }^{7}$ Peter Richmond, ${ }^{8}$ David Shaw, ${ }^{9}$ \\ David Gordon, ${ }^{10}$ Belinda Barton (iD) ${ }^{11}$
}

To cite: Marshall H, McMillan M, Wang B, et al. AMEND study protocol: a case-control study to assess the long-term impact of invasive meningococcal disease in Australian adolescents and young adults. BMJ Open 2019;9:e032583. doi:10.1136/ bmjopen-2019-032583

- Prepublication history for this paper is available online. To view these files, please visit the journal online (http://dx.doi org/10.1136/bmjopen-2019032583).

Received 26 June 2019 Revised 15 November 2019 Accepted 29 November 2019

Check for updates

(c) Author(s) (or their employer(s)) 2019. Re-use permitted under CC BY-NC. No commercial re-use. See rights and permissions. Published by BMJ.

For numbered affiliations see end of article.

Correspondence to Professor Helen Marshall; helen.marshall@adelaide.edu.au

\section{ABSTRACT}

Introduction Invasive meningococcal disease

(IMD) primarily causes disease in young children and adolescents and can cause long-term disability. Many countries are considering implementation of meningococcal $B$ and/or meningococcal ACWY vaccines to control meningococcal disease. Estimating the costeffectiveness of meningococcal vaccine programme is hampered due to a lack of good quality costing and burden of disease data. This study aims to address this evidence gap by assessing the clinical, physical, neurocognitive, economic and societal impact of IMD on adolescents and young adults.

Methods and analysis A case-control study of 64 participants with confirmed IMD (15-24 years 11 months at time of disease) and 64 control participants (17-34 years 11 months) will be conducted in Australia from 2016 to 2020. All participants will undergo a neurocognitive assessment, full medical examination, pure tone audiometry assessment and complete quality of life and behavioural questionnaires. Meningococcal cases will be assessed 2-10 years posthospitalisation and a subset of cases will be interviewed to explore in depth their experiences of IMD and its impact on their life. Primary outcome measures include general intellectual functioning from the Wechsler Adult Intelligence Scale and overall quality of life from the Health Utilities Index. Secondary outcome measures include academic achievement, executive functioning, behaviour, hearing, psychological and physical functioning. Outcome measures will be compared between cases and controls using independent t-tests or ORs, or if any significant confounders are identified, adjusted analyses (analysis of covariance or adjusted ORs) will be conducted. Thematic analysis will be used to analyse transcribed interviews and a costing model will be used to project lifetime costs.

Ethics and dissemination The Adolescent MENingococcal Disease (AMEND) study has been approved by the Human Research Ethics Committee of the Women's and Children's Health Network (HREC/14/WCHN/024). The results will be disseminated via peer-reviewed publications, conference presentations, study participants, and meningococcal and meningitis foundations.

Trial registration number NCT03798574.
Strengths and limitations of this study

- Generation of new evidence to inform vaccination policy for protecting adolescents and young adults from invasive meningococcal disease (IMD).

- Comprehensive assessment of the long-term effects of meningococcal disease on adolescents and young adults including clinical, neurocognitive and quality of life.

- National recruitment of adolescents and young adults with IMD ensures generalisability of the data to Australia and similar countries, such as New Zealand, Canada, the UK and the USA.

- There is the potential for selection bias to occur since the sampling of cases and controls is occurring using different methods.

- While data obtained from self-reported questionnaires and interviews provide valuable information about participants' perceptions of their own functioning, we cannot be confident that participants have provided accurate data free from recall or other bias.

\section{INTRODUCTION}

Invasive meningococcal disease (IMD) is one of the most common infectious causes of death in childhood in developed countries. ${ }^{1}$ Neisseria meningitidis, the cause of meningococcal disease, causes significant morbidity and mortality worldwide with approximately 500000-1 200000 cases and 50000-135000 deaths reported annually. ${ }^{23}$ IMD often manifests as septicaemia without or with meningitis $^{4}$ and can cause permanent sequelae, which may lead to significant disability in approximately $7.2 \%(4.3 \%-11.2 \%)$ of survivors. ${ }^{5}$

Survivors of IMD often experience a range of cognitive, psychosocial and physical sequelae that are mild to severe in nature and impact on their health-related quality of life (QoL). These sequelae occur both in 
the short-term and long-term post-IMD and have been reported in child and adult survivors. ${ }^{6} 7$ A large casecontrol study conducted in England found that around $10 \%$ of children approximately 3 years post-serogroup B IMD (mean age 6 years old at time of assessment) had a major disabling deficit. In addition, more than one-third of IMD cases $(36 \%)$ had one or more deficits in physical, cognitive and psychological functioning versus $15 \%$ of controls. ${ }^{7}$ However, while these deficits were relatively common, their impact on the QoL of children was not examined.

While meningococcal disease affects all age groups, the incidence of IMD peaks in the 0-4years, and 15-25years age groups in some countries, including Australia. ${ }^{8-10}$ To date, few studies have examined the long-term impact of IMD on adolescents and young adults (AYA) aged 15-25 years at the time of disease. This is an important transition period when AYA are learning to be responsible for their own medical care while experiencing many unmet healthcare needs and difficulties in accessing healthcare, ${ }^{11}$ as well as completing secondary schooling and planning for future tertiary options and/or employment. It is also a crucial developmental period associated with significant maturational changes in brain structure, neurochemistry and function, as well as changes in cognition and emotion, with increased risk-taking behaviour and onset of mental illness frequently occurring during this period. ${ }^{12}{ }^{13}$ The results from a study of young adult males (18-24 years at the time of IMD) conducted over 30 years ago indicated that $3-15$ years post-IMD, survivors reported significantly more symptoms of possible sequelae compared with the control group $(61 \%$ vs $20 \%) .{ }^{14}$ In addition, around $30 \%$ reported that the disease had affected their education or working capacity. ${ }^{14}$ In another study adolescents (15-19 years at time of IMD) who were followed up 18-36 months post-disease also reported poorer educational attainment, achieving fewer passes at high school and were twice as likely to have failed an examination in the last 12 months when compared with matched controls. ${ }^{6}$ Adolescent survivors also reported significantly poorer physical and mental health (ie, depression), as well as QoL when compared with controls. Disabling physical sequelae were identified in $57 \%$ of survivors and $5 \%$ required amputations. ${ }^{6}$

While IMD has a low incidence, it is associated with significant economic implications. A recent systematic review of studies that reported the financial costs associated with acute and long-term sequelae of IMD found that while IMD results in significant costs to healthcare systems, costing for long term and indirect costs are lacking. ${ }^{15}$ In addition, as the costs of hospitalisation and follow-up care reported in these studies were estimated only from a third-party payer's perspective, it is likely that the societal burden of IMD was underestimated. ${ }^{15}$ Further studies of indirect costs of IMD are imperative to estimate the total financial burden of IMD.

The health economic evaluation of meningococcal vaccine programmes has identified that further data on long-term sequelae would be beneficial. ${ }^{16}$ For vaccines against uncommon diseases, like IMD, the results of health economic evaluations can vary significantly depending on the parameter values used (eg, treatment costs, QoL losses of IMD) or on the basis of expert opinions. ${ }^{17} 18$ Cost-effectiveness analyses are challenging due to a paucity of data on disease burden, particularly a lack of data on long-term disability from IMD, making decisions on the introduction of meningococcal vaccination programme difficult. ${ }^{19}$

Although meningococcal vaccines are licensed in many countries, they are not necessarily publicly funded due to unknown or unfavourable cost-effectiveness analyses. ${ }^{20}$ Only the UK has introduced a national funded MenB vaccine programme that is provided for infants. Due to increasing incidence of meningococcal W IMD cases in the UK, a funded MenACWY vaccine programme has been introduced. ${ }^{21}$ In Australia, a MenACWY programme has been introduced for infants at 1 year of age and will be funded for adolescents 14-19 years of age from $2019 .{ }^{22}$ However, none of these programmes provides full protection against all meningococci, so disease will continue to be a burden in these age groups. Health authorities in several countries, such as Spain, are considering the introduction of MenB and MenACWY vaccines in their national immunisation programme, but detailed and contemporary data on the clinical benefit and long-term costs are not available, particularly for AYA. ${ }^{23}$

The findings from this study will assist in more robust data to inform policy as to whether meningococcal vaccines should be included in routine immunisation programme. Additionally, cost of illness studies can produce estimates of the real economic consequence over time of a specific disease and assist in understanding the importance of a particular health problem, particularly for a rare disease, such as meningococcal infection. ${ }^{24}$ Such studies can also be used to aid policy-makers to estimate cost savings and medical benefits in economic evaluations of healthcare interventions and to inform public health policies, such as funding priorities and immunisation programmes. ${ }^{25-27}$

In summary, survivors of IMD experience a range of mild to severe sequelae that impact on their QoL. The majority of studies to date have focused on the impact of IMD on childhood and very little is known about the impact of the disease on AYA. Given that this is a critical period, it is feasible that the impact of IMD disease during this time may be greater for AYA than younger children. In addition, there are no data on the longterm sequelae of IMD in survivors. Further research is warranted to understand the impact of sequelae of IMD on AYA, as well as the financial impact of the disease on individuals, their families and the healthcare system. Therefore, the overall aim of this study is to assess the physical, neurocognitive, economic and societal impact of IMD on AYA. 


\section{METHODS AND ANALYSIS}

\section{Study aims}

The primary aim of this study is to determine the longterm impact of IMD on general intellectual functioning and QoL of AYA. Secondary aims include (1) assessing the impact of IMD on neurocognitive (academic achievement, executive functioning, memory), psychological and physical functioning; (2) estimating the lifetime costs associated with survival following IMD and (3) comparing the burden of serogroup B IMD to non-B serogroup IMD. An exploratory aim is to examine the relationship between meningococcal serogroup type and disease severity/sequelae.

\section{Study design}

This is a multicentre, case-control, mixed-methods complementarity study.

\section{Study setting}

Identification of IMD cases will occur at each of the participating Australian hospitals (paediatric and adult) in Adelaide (Women's and Children's Hospital, Flinders Medical Centre, Royal Adelaide Hospital, Lyell McEwin Hospital and The Queen Elizabeth Hospital), Sydney (Children's Hospital at Westmead, Westmead Hospital and Royal Prince Alfred Hospital), Melbourne (Monash Children's Hospital, Monash Medical Centre and The Alfred Hospital) and Perth (Princess Margaret Hospital for Children and Sir Charles Gairdner Hospital). Of note, in Australia, children aged from birth to 16 years (and up to 18 years for pre-existing conditions) are admitted to a children's hospital for medical care.

\section{Study procedures}

Prospective cases will be identified by hospital staff who will conduct a daily surveillance of their hospital systems for patients who are admitted with suspected meningococcal infection as reported in their medical records and also access hospital separation data to identify any admissions coded with International Classification of Diseases (ICD) 10-A39.0 to A39.9 (as a primary or additional code) or coded $\mathrm{J} 15.8^{28}$ (see figure 1). After a diagnosis of IMD has been confirmed (please see inclusion criteria) by site medical staff, the treating hospital physician will provide patients with a study information sheet requesting that they contact the study investigators if they would like to participate.

Retrospective cases who were hospitalised 2-10 years ago with confirmed IMD will be identified by site staff from discharge coding, hospital medical records and/or an electronic database of patients' diagnoses maintained by the Infectious Diseases Department of the hospital. After a diagnosis of IMD has been confirmed by site medical staff, participants/parents will be mailed a study invitation letter inviting them to contact the study investigators if they have any questions or would like further information about the study. If they do not respond, three attempts will be made by the site staff to contact them by mail/phone.

Controls will be prospectively recruited by 'snowballing sampling' technique whereby enrolled IMD cases will be asked to distribute a study information sheet to their friends/acquaintances who are approximately the same age ${ }^{29}$ Potential controls who would like further information or would like to participate will contact the site staff by phone/email. Control participants may also be identified through community advertising or from research databases at each participating site. These databases are managed by participating hospitals and contain contact details of the people who have previously consented to be contacted about participating in future studies. The majority have been community participants of previous studies conducted at the hospital. Controls will be group matched by age and gender. Enrolment of participants commenced from 2016 and is expected to be completed by December 2020 .

\section{Eligibility \\ Inclusion criteria}

- Retrospective cases will be identified by using the following codes ICD 10-A39.0 to A39.9 ${ }^{28}$ or ICD 9-036 (as a primary or additional code) or coded $\mathrm{J} 15.8 .^{28}$ All IMD cases (retrospective and prospective) must have a confirmed infection by PCR, culture or cerebrospinal fluid with $N$. meningitidis of any serogroup, which will be verified by the site nurse or doctor.

- IMD cases must be aged 15-24 years 11 months inclusive at the time of IMD and currently hospitalised for IMD or recently separated (prospective); or hospitalised for IMD within the previous 2-10 years at the time of study assessments (retrospective).

- Controls will be aged 17-34 years 11 months at the time of assessment. The older age matches the age range of IMD cases at the time of their assessment which is $2-10$ years post-IMD.

\section{Exclusion criteria}

- Individuals who are not fluent with the English language since neurocognitive tests are only available in English.

- All participants with a known pre-existing intellectual disability and/or intracranial pathology (prior to hospitalisation for IMD cases).

- Control participants with a history of meningococcal disease.

\section{Physical, neurocognitive and hearing outcomes}

All participants will complete a neurocognitive and psychological assessment (see table 1) that will be conducted face to face by a psychologist and will take approximately 6 hours to complete. For all IMD cases (including those recruited prospectively), assessments will be conducted 2-10 years post-IMD admission. Psychologists conducting the assessments will be blinded (as far as possible) to case or control status. Participants will be advised not to 


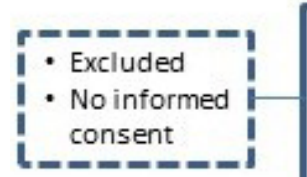

Prospective IMD cases $(n=30)$

- Patients hospitalised with suspected/confirmed IMD

- Invitation letter provided by site staff

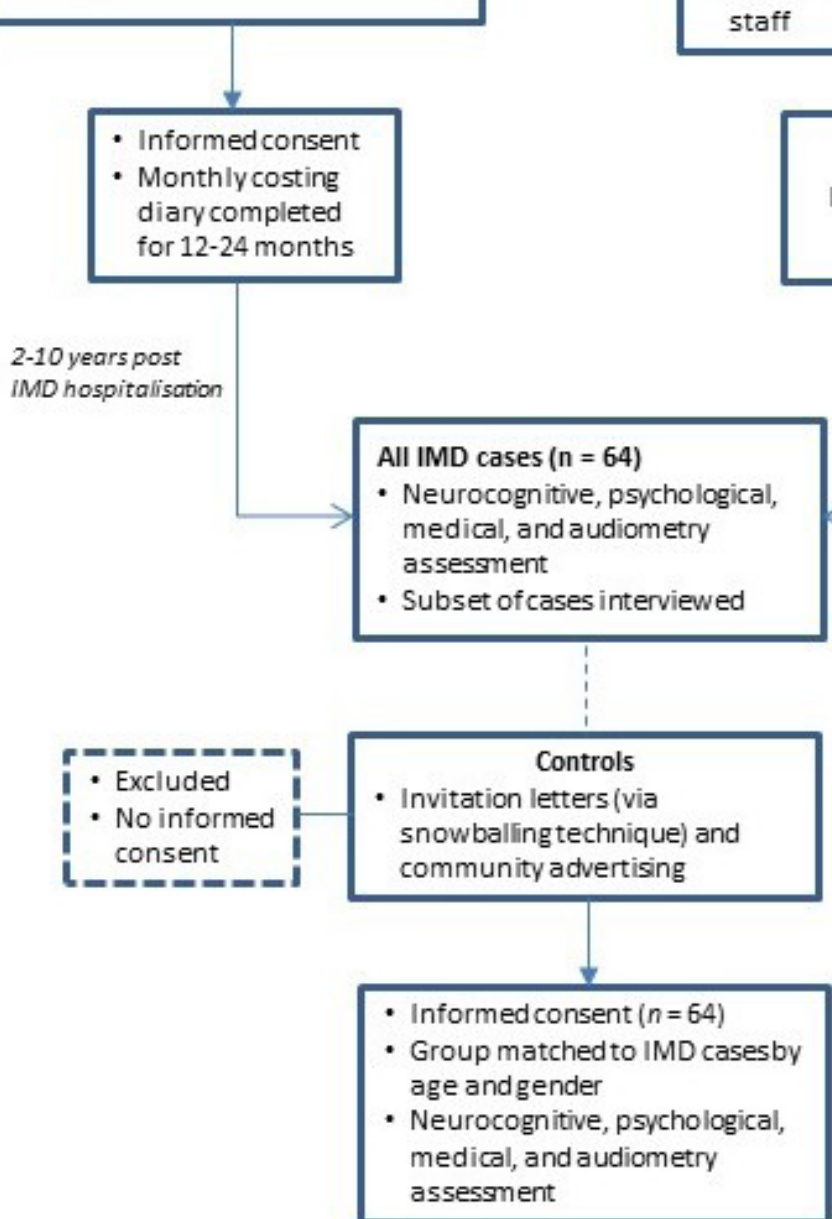

Retrospective IMD cases $(n=34)$

- Patients hospitalised with confirmed IMD 2-10 years ago identified through medical records

- Invitation letter mailed out by site staff

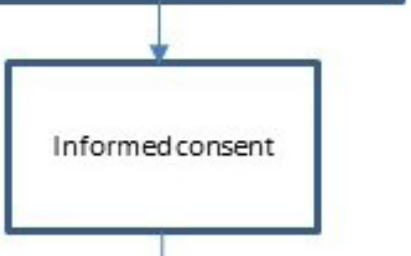

disclose their case/control status to the psychologist. On completion of all outcome measures participants will be provided with a AUD $\$ 150$ voucher to cover any costs associated with travelling and their time in completing the assessments.

\section{Primary outcome measures}

Intellectual functioning will be measured by the Full Scale IQ score obtained from the Wechsler Adult Intelligence Scale-Fourth Edition (WAIS-IV), ${ }^{30}$ a widely used and standardised test of intelligence.

QoL will be measured by the overall multiattribute health utility score obtained from the Health Utilities Index Mark 3 (HUI3)-15Q self-report. ${ }^{31}$ The HUI3 consists of 15 items assessing the following domains: vision, hearing, speech, cognition, pain, emotion, ambulation and dexterity. The HUI has been used in previous IMD studies including children (16 years) approximately 5 months post-IMD (group B) ${ }^{32}$ and survivors of meningococcal septic shock. ${ }^{33}$

\section{Secondary outcome measures}

\section{Neurocognitive and psychological outcomes}

Standardised psychometric measures assessing academic achievement, executive and memory (verbal and visual) functioning of all participants will be administered by a psychologist (table 1). Questionnaires assessing attention, executive functioning, behaviour and psychological problems will also be completed by participants and/or parents (table 1). Participants will undergo a structured diagnostic interview conducted by the site psychologist to screen for psychiatric disorders (table 1). On completion, all participants will receive a follow-up phone call/feedback from the psychologist and a brief summary report of their neurocognitive results.

\section{Medical and audiometry examination}

Each participant will undergo a full medical examination conducted by the site physician using the International Classification of Functioning, Disability and Health tool to assess for the presence of body function/structure 
Table 1 Neurocognitive and psychological outcomes

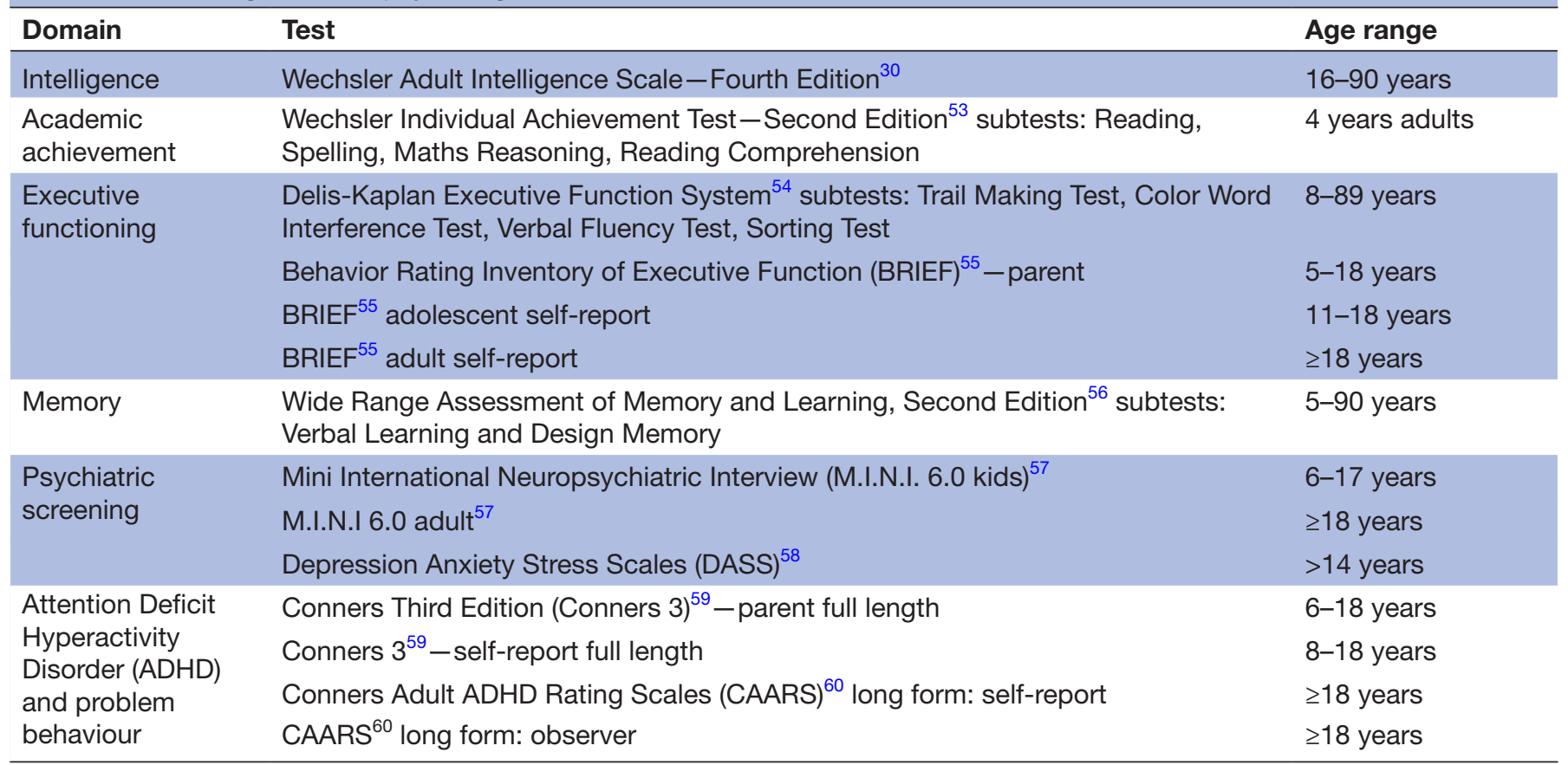

impairments, restrictions in physical activities and participation. ${ }^{34}$ A pure tone audiometry will be conducted and hearing will be classified as no impairment (0) to profound $(5) .{ }^{35}$

\section{QOL and carer experience}

All participants will complete the the 5-level EQ-5D (EQ-5D-5L) to measure their health status, which will be used to calculate quality-adjusted life years lost (table 2). For those participants with a disability, the primary caregiver and other family members living in the same household will be invited to complete questionnaires assessing their well-being and carer experience (table 2). All questionnaires shown in table 2 have been used in previous meningococcal studies. $^{32} 33$ 36-40

\section{Socioeconomic status}

Socioeconomic status (SES) of participants will be estimated using the index of relative socioeconomic advantage and disadvantage, which ranks geographical areas in terms of their socioeconomic advantage and disadvantage. ${ }^{41}$ The lowest $10 \%$ of areas are ranked a decile of 1 and the highest $10 \%$ are ranked a decile of 10 .

\section{Clinical information for IMD cases only}

A standardised data collection sheet will be completed to capture information on clinical disease, management, complications, outcomes and sequelae for IMD cases. Data on age, gender, indigenous status, comorbidities, social demographic (eg, residence areas, postcodes), length of admission and outcome will be recorded by medical or nursing staff at each participating hospital. In addition, signed informed consent will be obtained from participants to access health databases including Medicare (publicly funded universal healthcare system); the Australian Government Pharmaceutical Benefits Scheme $(\mathrm{PBS})^{42}$ national programme, which subsidises the cost of a wide range of prescribed medicines for all Australians; and general practitioner/specialist clinical records. IMD cases recruited prospectively will be asked to complete monthly diary cards for at least 12 months and up to 24

Table 2 Quality of life (QoL) and carer questionnaires

\begin{tabular}{llcc}
\hline Domain & Test & Age range & Completed by \\
\hline Overall QoL & ICEpop CAPability measure for adults ${ }^{61}$ & $\begin{array}{c}\geq 18 \text { years } \\
\text { Parent and other } \\
\text { family members }\end{array}$ \\
Care-related QoL & Carer Experience Scale $\left(6\right.$ questions) ${ }^{62}$ & $\geq 18$ years & Primary caregiver \\
Health-related QoL & $\begin{array}{l}\text { Health Utilities Index Mark } 3-15 Q^{31} \text { domains: vision, } \\
\text { hearing, speech, cognition, pain, emotion, ambulation }\end{array}$ & Participant & \\
& and dexterity.
\end{tabular}

Health status to calculate qualityadjusted life years lost self-care, usual activities, pain/discomfort, anxiety/ depression. 
months depending on the time of enrolment to obtain details of any medical follow-up and progress in relation to sequelae and associated direct non-healthcare and indirect costs. The site study coordinator will phone/ email participants monthly to check that the diary is being completed and returned to the site investigator via provided self-addressed envelopes/email.

\section{Qualitative data}

\section{Semistructured interviews for IMD cases only}

To obtain more detailed information about the impact of IMD, in-depth interviews on a subset of IMD cases will be conducted until thematic saturation. The interview will be semistructured and consist of a series of questions (eg, can you tell me about the symptoms and treatment you received for IMD; does IMD impact on your daily life, if yes, how); however, the interviewer will be trained in techniques to allow the interview to be flexible, to generate new questions during the interview, to probe for details and discuss issues that arise during the interview. Interviews will be completed face to face, although if this is not possible, they will be performed over the phone. Interviews will be completed 2-10 years postdiagnosis of IMD and audio recorded.

\section{Adverse event monitoring}

The study-related adverse event (AE) reporting period commences when the participant provides informed consent and continues until study participation is complete. An expected AE of the study is that a participant becomes distressed when completing the assessments and/or interview (IMD cases only). All AEs will be reported to the relevant Human Research Ethics Committee. For all AEs, the site investigator will be required to assess and record the causal relationship. Sufficient information will be obtained by the site investigator to determine the causality of each AE. The investigator will be required to follow up AEs until the event and/or its sequelae resolve or stabilise at a level acceptable to the investigator. An investigator's causality assessment is the determination of whether there exists a reasonable possibility that any study processes caused or contributed to an $\mathrm{AE}$.

\section{Sample size}

The primary outcomes are Full Scale IQ (WAIS-IV) and QoL (HUI-3 overall) scores. In a large IMD case-control study of children, there was a difference of 7.5 Full Scale IQ points between cases and controls matched by age and gender, ${ }^{7}$ an estimated medium effect size (Cohen's $\mathrm{d}=0.50$ ). In the same study, ${ }^{7}$ an unmatched comparison of IMD cases to controls indicated a difference of 7.4 Full Scale IQ points also representing a medium effect size. In a study investigating the QoL of mainly childhood survivors (median age 14.5 years, age range 5-31 years) approximately 10 years postintensive care discharge for IMD, HUI-3 overall scores were significantly lower by 0.11 when compared with normative data, ${ }^{33}$ representing a medium effect size (Cohen's d=0.56). Therefore, based on these previous studies, we have estimated a medium effect size (Cohen's d=0.50) between cases and controls. To detect a medium effect size between groups using an independent t-test, with $80 \%$ power, two-tailed significance of $0.05,64$ ( $+10 \%$ for lost to follow-up) participants are required in each group. ${ }^{43}$

\section{Statistical analysis}

Quantitative analyses

Descriptive statistics will be reported. Continuous variables will be compared between cases and controls using independent t-tests. Categorical variables will be compared between groups using tests of $\mathrm{X}^{2}$ or ORs $(95 \% \mathrm{CI})$. However, if any significant confounders (eg, age, gender, SES) are identified then an adjusted analysis using analysis of covariance and adjusted ORs (95\% CI) will be conducted. All tests will be two tailed with the Benjamini-Hochberg method applied to reduce the False Discovery Rate (FDR) by controlling for multiple hypotheses testing. ${ }^{44}$ For continuous variables, effect sizes (Cohen's d) will also be calculated.

For neurocognitive outcomes, the level of impairment will also be classified by the number of SD below the normative mean (mild: 1.0-1.9 SD below, moderate: 2.0-2.9 SD and severe: $\geq 3.0 \mathrm{SD}$ ). Differences in medical examination and audiometry findings including the type and frequency of hearing impairments in cases and controls will be reported descriptively. Definitions of major and minor sequelae will be classified using WHO Global Burden of Disease ${ }^{45}$ and a systematic review/metaanalysis of disabling sequelae from bacterial meningitis. ${ }^{5}$ This classification of sequelae was used in a previous IMD study. ${ }^{7}$ Major sequelae are defined as cognitive impairment (Full Scale $<70$ ), bilateral sensorineural hearing loss ( $\geq 40 \mathrm{~dB}$ ), seizures (any type), disabling motor impairment (eg, amputation of part of a limb or more than one digit), significant visual loss or major communication impairment (unintelligible speech or cannot understand speech). Minor sequelae are defined as other cognitive, hearing, motor, visual, communication impairments and psychological disorders. ORs (95\% CI) for the occurrence any minor, any major and all sequelae will be calculated.

An exploratory multiple linear regression to identify predictors of QoL of IMD participants will be conducted. Potential predictors include Full Scale IQ time from IMD hospitalisation to study assessment, presence/absence of major sequelae and psychological functioning. If there are sufficient serogroup B IMD cases, their outcomes will be compared with non-serogroup B IMD cases using the same analyses as mentioned above for continuous and categorical variables.

In addition, to assess the impact of potential correlation between participants in the same hospital, we will conduct a sensitivity analysis to determine the effect of any potential clustering on the outcomes and the conclusions of the study and to estimate correlation within clusters, for example, using generalised estimating equations. 


\section{Health economic analyses}

We anticipate obtaining consent to access health databases including Medicare and the PBS from all IMD cases enrolled $(n=64)$. Direct medical costs will be based on routinely collected data describing the type and frequency of inpatient separations obtained from state health databases for hospital admissions. The cost of outpatient services (eg, visits to primary care physicians) and pharmaceuticals will be derived from Medicare including PBS.

Patient's monthly diary cards completed by prospective patients (estimated $n=30$ ) will be used to estimate other direct costs, such as out of pocket costs, health services, which are not covered by Medicare (eg, ambulance services) and copayments (eg, on pharmaceuticals), as well as direct non-medical costs, such as travel costs and time spent travelling to medical appointments, and indirect costs due to cessation or reduction of workforce activity (productivity).

A micro-costing (bottom-up) approach, which provides detailed cost information, will be used to estimate costs associated with IMD from the healthcare system and societal perspectives. A costing model will be developed to estimate lifetime costs associated with IMD, taking into account different discount rates (ie, annual rates of $3.5 \%$ or $5 \%) .{ }^{46}$ By using the micro-costing approach, resources used at the individual level will be assessed and costs of individual patients will be aggregated. The micro-costing approach reflects the true costs to deliver care to the individual patient . ${ }^{47}$ The bottom-up approach, which highly depends on availability of data on treatment costs or productivity losses, can provide more detailed information for analysis and stratification than top-down (population-based) approach. By using the top-down method, total healthcare costs would be disaggregated, and a relevant portion of the total costs would be allocated to a specific disease. ${ }^{47}$ A decision analytic model (eg, Markov model with yearly cycles) will be built. The model structure will include health states and transitions between them representing the type of care required with death as an absorbing state. Relevant cost estimates per cycle will then be attached to states included in the model. A hypothetical birth cohort will be followed over a 100-year time horizon. Health states, probabilities of health states and costing parameters will be obtained from a variety of sources including the present study and/ or published literature. The best available evidence will be used to inform model structure and inputs.

In addition to reporting the base case analysis, the model developed will be used to undertake sensitivity analysis over a range of uncertain parameters to inform the likely impact of using alternative values.

\section{Qualitative analyses}

Interviews will be transcribed verbatim and analysed using iterative thematic analysis techniques to enable an understanding of the participant's experiences of IMD in particular, details of their hospitalisation and treatment, the impact of IMD on their daily life after being discharged and currently and details of any support (eg, social, healthcare professionals) that they have received. Similar to the methods used previously, ${ }^{48}$ interview transcripts will be subjected to coding by one investigator. A second investigator will code transcripts independently, and then both investigators will meet to discuss their analysis. This iterative process will allow movement between data collection and analysis as codes are interpreted and themes generated. Transcripts will be read and reread and initial codes assigned based on the language used by the participants themselves. Discussion between researchers, coding notes and memos will be used to ensure consistency in the coding framework. Initial themes will be identified by discussion between the researchers and matrixes, grids and tables will be used to visualise the relationship between the themes and the experiences of each of the participants. Qualitative findings will be used to complement ${ }^{49}$ quantitative findings. For example, major sequelae may impact on the QoL participants and interviews will provide further richness and understanding on how the sequelae impacts on their life.

\section{Patient and public involvement}

The research question was developed in response to policy advisors identification of the evidence gap in understanding the long-term impact of meningococcal disease on survivors. Assistance in study processes has been provided by meningococcal and meningitis support groups in Australia.

\section{Data management and confidentiality}

Identifying documents will be maintained at each participating site in locked cabinets and offices. Data management will be coordinated and overseen by the site principal investigator (PI) at the Women's and Children's Health Network, Adelaide. Quantitative data collected during the study will be entered by site staff into an online (Research Electronic Data Capture [REDcap]) database in a reidentifiable manner. The electronic database is username and password protected and located on the server at the University of Adelaide. Except for the University of Adelaide staff who will be analysing the data, all other site investigators can only access and view data from their own site. Following data analysis, the data will be deleted from REDcap and a deidentified password-protected dataset will be stored on the University of Adelaide server and deleted after 30 years.

Reidentifiable data are identifiable only at the recruiting study site where a master participant code list will be retained by the site investigator. The list will be stored electronically on their computer that is password protected and only accessible to them. Information published from this study will not identify any participants involved in this study.

\section{Ethics and dissemination}

Participants who are at least 18 years of age will be approached and the study discussed with them. If they 
agree to participate, an information sheet will be provided with an opportunity to discuss the study with the study team and a consent form will be signed by the participant. If the participant is 17 years of age or less, assent will be obtained and the parent/guardian will provide informed signed consent. A second consent to release of Medicare and/or PBS claims information form is also required to be signed prior to release of information from the Department of Human Services. Detection of neurocognitive impairments and/or elevated psychological symptoms (eg, symptoms of depression) may be upsetting to participants and their families. We will facilitate referral for follow-up with their family physician and/or psychologist where appropriate with consent of the participant.

The results will be disseminated via peer-reviewed publications and conference presentations, and a summary of the findings will be provided to study participants, the wider community and meningococcal support foundations. The results may also be reported on websites (eg, hospitals, foundations) and in the media including television, radio and print media.

\section{Significance}

This study is being conducted at a time when, increasingly, public health strategies are subject to consideration of the relative economic cost of the proposed strategy. Our study will contribute robust data to assess the societal cost of disability from infectious disease by examining the most common infectious cause of death in AYA in Australia, IMD.

The strengths of our study include the use of both objective and subjective standardised measures to determine the long-term outcomes and disability experienced by IMD survivors, national recruitment of IMD cases and only those who are AYA. Some IMD survivors may be less likely to have resources to attend study locations and/ or may come from rural settings and have lower SES. However, we have attempted to ameliorate any participation bias by providing travel reimbursement for participants. For patients with severe disabilities, their health conditions and inconvenience may prevent participation in the study.

This national study will include data from four states of Australia. These findings will have global significance as other countries are currently considering introduction of meningococcal vaccines in their national immunisation programmes. The UK Joint Committee on Vaccination and Immunisation initially concluded that an infant MenB vaccine programme would not be cost-effective and recommended not funding Men B immunisation. ${ }^{18}$ However, negotiations with the manufacturer resulted in an agreed price and a programme commenced in 2015. ${ }^{21}$ In Australia, the Pharmaceutical Benefits Advisory Committee rejected including MenB vaccine on the publicly funded national immunisation schedule on three occasions (2013-2015) due to uncertainties and assumptions used in the cost-effectiveness model. ${ }^{46}$ 50-52 As only limited data exist globally on the long-term burden of IMD our study will provide comprehensive data on the impact of IMD on AYA survivors, which can further inform cost-effectiveness estimates, particularly for adolescent programme.

\section{CONCLUSION}

Australia has limited outcome data for patients who survive IMD and little is known about the impact the disease has on the life of AYA survivors. The results from this study will provide the comprehensive data required to understand the impact of IMD in young people, as well as to assess the long-term health and financial implications for the individual, their families and the healthcare system. These data are essential for cost-effectiveness estimates for countries considering the introduction of this uncommon but potentially life-threatening and disabling infection.

\section{Author affiliations}

${ }^{1}$ Vaccinology and Immunology Research Trials Unit, Women's and Children's Health Network, Adelaide, South Australia, Australia

${ }^{2}$ Robinson Research Institute and Adelaide Medical School, University of Adelaide, Adelaide, South Australia, Australia

${ }^{3}$ The Discipline of Child and Adolescent Health, The Children's Hospital at Westmead, University of Sydney Medical School, Westmead, New South Wales, Australia

${ }^{4}$ National Centre for Immunisation Research and Surveillance (NCIRS), The Children's Hospital at Westmead, Sydney, New South Wales, Australia

${ }^{5}$ School of Public Health, Univeristy of Adelaide, Adelaide, South Australia, Australia

${ }^{6}$ Department of Paediatrics, Royal Children's Hospital, Melbourne, Victoria, Australia ${ }^{7}$ Department of Infectious Diseases, Perth Children's Hospital, Perth, Western Australia, Australia

${ }^{8}$ School of Medicine, University of Western Australia and Wesfarmers Centre of Vaccines and Infectious Diseases, Telethon Kid's Institute, Perth, Western Australia, Australia

${ }^{9}$ Infectious Diseases Service, Central Health Service, SA Health, Adelaide, South Australia, Australia

${ }^{10}$ Microbiology and Infectious Diseases, Flinders Medical Centre, Bedford Park, South Australia, Australia

${ }^{11}$ Children's Hospital Education Research Institute (CHERI), The Children's Hospital at Westmead, Westmead, New South Wales, Australia

Contributors HM is the lead investigator on the AMEND study and designed the study protocol with BB, RB, PR, CCB, MM, BW, JB, DS, DG and HA. All the authors participated in the drafting and revision of the manuscript and approved the final version and agree to be accountable for the contents and integrity of this manuscript.

Funding Funding for this study was provided by Pfizer. Pfizer was provided the opportunity to review a preliminary version of this manuscript for factual accuracy but the authors are solely responsible for final content and interpretation.

Disclaimer The funder will not be involved in study processes, analysis or interpretation of the data. The authors received no financial support or other form of compensation related to the development of the manuscript.

Competing interests HM is supported by an NHMRC CDF APP1084951 and is a member of the Australian Technical Advisory Group on Immunisation, Australian Government. HM is an investigator on vaccine trials sponsored by Industry (GSK, Novavax, Pfizer). HM and MM's institution receives funding for investigator-led studies from Industry (Pfizer, GSK). HM and MM receive no personal payments from Industry. CCB is supported by an NHMRC CDF APP1111596 and is a member of the Australian Technical Advisory Group on Immunisation, Australian Government. PR is an investigator on vaccine trials sponsored by Industry (GSK, Novavax, Pfizer). PR's institution receives funding for investigator-led studies from Industry (Pfizer, GSK, CSL). PR has been a member of scientific vaccine advisory boards for Industry (Pfizer, GSK, Sanofi) but has not received any personal payments from Industry. DG 
has received research funding support from Sanofi-Pasteur for an unrelated study and has been an investigator on non-meningococcal vaccine studies sponsored by Industry (Sanofi-Pasteur). RB has received funding from Baxter, CSL/Seqirus, GSK, Merck, Novartis, Pfizer, Roche, Romark and Sanofi Pasteur for the conduct of sponsored research, travel to present at conferences or consultancy work; all funding received is directed to research accounts at The Children's Hospital at Westmead. HA is a member of the Australian Evaluation Sub-Committee (ESC) of the Medical Services Advisory Committee (MSAC).

\section{Patient consent for publication Not required.}

Ethics approval The study has been approved by the Women's and Children's Health Network Human Research Ethics Committee. Governance and ethics approval has also been obtained at all other affiliated sites.

Provenance and peer review Not commissioned; externally peer reviewed.

Open access This is an open access article distributed in accordance with the Creative Commons Attribution Non Commercial (CC BY-NC 4.0) license, which permits others to distribute, remix, adapt, build upon this work non-commercially, and license their derivative works on different terms, provided the original work is properly cited, appropriate credit is given, any changes made indicated, and the use is non-commercial. See: http://creativecommons.org/licenses/by-nc/4.0/.

\section{ORCID iDs}

Helen Marshall http://orcid.org/0000-0003-2521-5166

Mark McMillan http://orcid.org/0000-0002-6490-7707

Belinda Barton http://orcid.org/0000-0002-3157-2031

\section{REFERENCES}

1 Hart CA, Thomson APJ. Meningococcal disease and its management in children. BMJ 2006;333:685-90.

2 Chang Q, Tzeng Y-L, Stephens DS. Meningococcal disease: changes in epidemiology and prevention. Clin Epidemiol 2012;4:237-45.

3 Jafri RZ, Ali A, Messonnier NE, et al. Global epidemiology of invasive meningococcal disease. Popul Health Metr 2013;11:17.

4 Baker CJ. Prevention of meningococcal infection in the United States: current recommendations and future considerations. $J$ Adolesc Health 2016;59:S29-37.

5 Edmond K, Clark A, Korczak VS, et al. Global and regional risk of disabling sequelae from bacterial meningitis: a systematic review and meta-analysis. Lancet Infect Dis 2010;10:317-28.

6 Borg J, Christie D, Coen PG, et al. Outcomes of meningococcal disease in adolescence: prospective, matched-cohort study. Pediatrics 2009;123:e502-9.

7 Viner RM, Booy R, Johnson $\mathrm{H}$, et al. Outcomes of invasive meningococcal serogroup $B$ disease in children and adolescents (MOSAIC): a case-control study. Lancet Neurology 2012;11:774-83.

8 Lahra M, Enriquez R. Australian meningococcal surveillance programme annual report, 2014, 2016. Available: http://www.health. gov.au/internet/main/publishing.nsf/Content/cda-pubs-annlrptmenganrep.htm [Accessed 16 Aug 2016].

9 Davis KL, Bell TJ, Miller JM, et al. Hospital costs, length of stay and mortality associated with childhood, adolescent and young adult meningococcal disease in the US. Appl Health Econ Health Policy 2011;9:197-207.

10 Pelton SI. The global evolution of meningococcal epidemiology following the introduction of meningococcal vaccines. J Adolesc Health 2016;59:S3-11.

11 Patton GC, Sawyer SM, Santelli JS, et al. Our future: a Lancet commission on adolescent health and wellbeing. Lancet 2016;387:2423-78.

12 Keshavan MS, Giedd J, Lau JYF, et al. Changes in the adolescent brain and the pathophysiology of psychotic disorders. Lancet Psychiatry 2014;1:549-58.

13 Spear LP. The adolescent brain and age-related behavioral manifestations. Neurosci Biobehav Rev 2000;24:417-63.

14 Sander J, Bay D, Gedde-Dahl TW, et al. Late sequelae after meningococcal disease. A controlled study in young men. NIPH Ann 1984;7:3-11.

15 Wang B, Santoreneos R, Afzali $\mathrm{H}$, et al. Costs of invasive meningococcal disease: a global systematic review. Pharmacoeconomics 2018;36:1201-22.

16 Borrow R, Alarcón P, Carlos J, et al. The global meningococcal initiative: global epidemiology, the impact of vaccines on meningococcal disease and the importance of herd protection. Expert Rev Vaccines 2017;16:313-28.
17 Jit M, Hutubessy R. Methodological challenges to economic evaluations of vaccines: is a common approach still possible? Appl Health Econ Health Policy 2016;14:245-52.

18 Joint Committee on Vaccination and Immunisation (JCVI). JCVI interim position statement on use of Bexsero $₫$ meningococcal $B$ vaccine in the UK 2013. Vol 2018. UK: Department of Health and Social Care, 2013.

19 Getsios D, Caro I, El-Hadi W, et al. Assessing the economics of vaccination for Neisseria meningitidis in industrialized nations: a review and recommendations for further research. Int J Technol Assess Health Care 2004;20:280-8.

20 European Centre for Disease Prevention and Control. Expert opinion on the introduction of the meningococcal $B(4 C M e n B)$ vaccine in the EU/EEA. Stockholm: ECDC, 2017.

21 Ladhani SN, Ramsay M, Borrow R, et al. Enter B and W: two new meningococcal vaccine programmes launched. Arch Dis Child 2016;101:91-5.

22 Australian Technical Advisory Group on Immunisation. National immunisation program schedule. Vol 2019. 10th edn. Canberra: Australian Government Department of Health, 2018.

23 Pharmaceutical Benefits Advisory Committee. Meningococcal polysaccharide conjugate vaccine serogroups $A, C, W-135$ and Y(adolescents): Pre-filled syringe, 0.5mL; Nimenrix $®$ Pharmaceutical Benefits Scheme. Canberra: Department of Health, Australian Government, 2018.

24 Larg A, Moss JR. Cost-of-illness studies: a guide to critical evaluation. Pharmacoeconomics 2011;29:653-71.

25 Byford S, Torgerson DJ, Raftery J. Economic note: cost of illness studies. BMJ 2000;320:1335.

26 Durand-Zaleski I. Why cost-of-illness studies are important and inform policy. Vasc Med 2008;13:251-3.

27 Chisholm D, Saxena S, Van Ommeren M. Dollars, DALYs and decisions: economic aspects of the mental health system. Geneva: World Health Organization, 2006.

28 International statistical classification of diseases and related health problems. 10th edn. Geneva: World Health Organisation, 2018.

29 The SAGE encyclopedia of social science research methods. Thousand Oaks, California: Sage Publications, Inc, 2004.

30 Wechsler D. Wechsler adult intelligence Scale-4th edition (WAIS-IV) Australian and New Zealand adaptation. Sydney, NSW: Pearson, 2008.

31 Furlong WJ, Feeny DH, Torrance GW. Health utilities index® mark 2 and mark 3 (hui2/3) 15-item questionnaire for Self-administered, selfassessed "four week" health status assessment. Dundas ON Canada: Health Utilities Inc, 2016.

32 Kennedy ITR, van Hoek AJ, Ribeiro S, et al. Short-term changes in the health state of children with group B meningococcal disease: a prospective, national cohort study. PLoS One 2017;12:e0177082.

33 Buysse CMP, Raat $\mathrm{H}$, Hazelzet JA, et al. Long-term health status in childhood survivors of meningococcal septic shock. Arch Pediatr Adolesc Med 2008;162:1036-41.

34 Kostanjsek N. Use of the International classification of functioning, disability and health (ICF) as a conceptual framework and common language for disability statistics and health information systems. BMC Public Health 2011;11:S3.

35 Mathers C, Smith A, Concha M. Global burden of hearing loss in the year 2000. Global burden of Disease 2000;18:1-30.

36 Al-Janabi H, Manca A, Coast J. Predicting carer health effects for use in economic evaluation. PLoS One 2017;12:e0184886.

37 Al-Janabi H, Van Exel J, Brouwer W, et al. Measuring health Spillovers for economic evaluation: a case study in meningitis. Health Econ 2016;25:1529-44.

38 Erickson LJ, De Wals P, McMahon J, et al. Complications of meningococcal disease in college students. Clin Infect Dis 2001;33:737-9.

39 Koomen I, Grobbee DE, Jennekens-Schinkel A, et al. Parental perception of educational, behavioural and general health problems in school-age survivors of bacterial meningitis. Acta Paediatr 2003;92:177-85.

40 Oostenbrink R, A Moll HA, Essink-Bot M-L. The EQ-5D and the health Utilities index for permanent sequelae after meningitis: a head-to-head comparison. J Clin Epidemiol 2002;55:791-9.

41 Statistical Local Area. Table 2: socio-economic indexes for areas (SEIFA), data cube: Excel spreadsheet, cat. No. 2033.0.55.001, 2011. Available: http://www.abs.gov.au/ausstats/abs@.nsf/DetailsPage/ 2033.0.55.0012011? OpenDocument [Accessed 21 Sep 2017].

42 Pharmaceutical benefits scheme. Available: www.pbs.gov.au [Accessed 10 Nov 2019].

43 Faul F, Erdfelder E, Buchner A, et al. Statistical power analyses using G*Power 3.1: tests for correlation and regression analyses. Behav Res Methods 2009;41:1149-60. 
44 Benjamini Y, Hochberg Y. Controlling the false discovery rate: a practical and powerful approach to multiple testing. $J$ R Stat Soc Series B 1995;57:289-300.

45 Murray CJ, Lopez AD, Organization WH. The global burden of disease: a comprehensive assessment of mortality and disability from diseases, injuries, and risk factors in 1990 and projected to 2020: summary 1996.

46 Pharmaceutical Benefits Advisory Committee. Guidelines for preparing submissions to the pharmaceutical benefits Advisory Committee (version 5.0). Vol 2018. Canberra: Australian Government Department of Health and Ageing, 2016.

47 Frick KD. Microcosting quantity data collection methods. Med Care 2009;47:S76-81.

48 Collins J, Alona I, Tooher R, et al. Increased awareness and health care provider endorsement is required to encourage pregnant women to be vaccinated. Hum Vaccin Immunother 2014;10:2922-9.

49 Tariq S, Woodman J. Using mixed methods in health research. JRSM Short Rep 2013;4:2042533313479197.

50 Pharmaceutical Benefits Advisory Committee. Multicomponent Meningococcal Group B Vaccine, $0.5 \mathrm{~mL}$, injection, prefilled syringe, Bexsero ${ }^{\circledR}$ - November 2013. Vol 2018. Canberra, Australia: Pharmaceutical Benefits Scheme, 2013.

51 Pharmaceutical Benefits Advisory Committee. Multicomponent Meningococcal Group B Vaccine, $0.5 \mathrm{~mL}$, injection, prefilled syringe, Bexsero® - July 2014. Vol 2018. Canberra, Australia: Australian Government Department of Health, 2014.

52 Pharmaceutical Benefits Advisory Committee. Multicomponent Meningococcal Group B Vaccine (4CmenB); $0.5 \mathrm{~mL}$ suspension for injection pre-filled syringe; Bexsero® - July 2015. Vol 2018. Canberra, Australia: Pharmaceutical Benefits Scheme, 2015.

53 Wechsler D. Wechsler Individual Achievement Test- Second Edition (WIAT-II). London, UK: Psychological Corporation, 2005.

54 Delis D, Kaplan E C, Kramer JH. Delis-Kaplan Executive Function System. San Antonio, TX: The Psychological Corporation, 2001.

55 Gioia G, Isquith P, Guy S, et al. Behavior Rating Inventory of Executive Function (BRIEF). Lutz, Florida: Psychological Assessment Resources, 2000.

56 Sheslow D, Adams W. Wide Range Assessment of Memory and Learning Second Edition (WRAML2). Bloomington: Pearson, 2003.

57 Sheehan DV, Lecrubier Y, Sheehan KH, et al. The Mini-International neuropsychiatric interview (M.I.N.I.): the development and validation of a structured diagnostic psychiatric interview for DSM-IV and ICD10. J Clin Psychiatry 1998;59:22-33. quiz 34-57.

58 Psychology Foundation of Australia. Depression anxiety stress scales (DASS). Psychology Department, UNSW: UNSW, 2014.

59 Conners KED, Sparrow E. Conners 3rd edition (Conners) manual. Canada: Multi-Health Systems Inc, 2009.

60 Conners KED, Sparrow E. Conners' Adult ADHD Rating Scales. Canada: Multi-Health Systems Inc, 2002.

61 Al-Janabi H, Peters TJ, Brazier J, et al. An investigation of the construct validity of the ICECAP-A capability measure. Qual Life Res 2013;22:1831-40.

62 Al-Janabi H, Flynn TN, Coast J. Estimation of a preference-based carer experience scale. Med Decis Making 2011;31:458-68.

63 EQ-5D-5L Health Questionnaire. English version for Australia. Netherlands: EuroQol Group EQ-5D, 2009. 